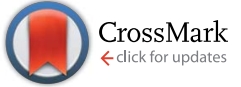

Cite this: RSC Adv., 2017, 7, 12407

Received 11th January 2017

Accepted 14th February 2017

DOI: $10.1039 / \mathrm{c} 7 \mathrm{ra00417f}$

rsc.li/rsc-advances

\section{Franck-Condon simulation for unraveling vibronic origin in solvent enhanced absorption and fluorescence spectra of rubrene $\uparrow$}

\author{
Ying Hu, tab $^{\text {ab }}$ Chen-Wen Wang, $\star^{\text {b }}$ Chaoyuan Zhu, ${ }^{* a b}$ Fenglong Gu*a \\ and Sheng-Hsien Lin ${ }^{b}$
}

Quantum chemistry calculations at the level of (TD)-DFT plus PCM solvent models are employed for analyzing potential energy surfaces and as a result two local minima with $D_{2}$, two local minima with $C_{2 H}$, and one second-order transition state with $D_{2 H}$ group symmetry are found in both ground $S_{0}$ and excited-state $S_{1}$ potential energy surfaces. Simulated vibronic coupling distributions indicate that only second-order transition states with $D_{2 \mathrm{H}}$ group symmetry are responsible for observed absorption and fluorescence spectra of rubrene and vibrational normal-motions related with atoms on the aromatic backbone are active for vibronic spectra. The Stokes shift $1120 \mathrm{~cm}^{-1}\left(820 \mathrm{~cm}^{-1}\right)$ and vibronic-band peak positions in both absorption and fluorescence spectra in non-polar benzene (polar cyclohexane) solvent are well reproduced within the conventional Franck-Condon simulation. By adding damped oscillator correction to Franck-Condon simulation, solvent enhanced vibronic-band intensities and shapes are well reproduced. Four (three) normal modes with vibration frequency around $1550 \mathrm{~cm}^{-1}\left(1350 \mathrm{~cm}^{-1}\right)$ related to ring wagging plus $\mathrm{CC}$ stretching and $\mathrm{CH}$ bend motions on the backbone are actually interpreted for solvent enhanced absorption (fluorescence) spectra of rubrene in benzene and cyclohexane solutions.

\section{Introduction}

Franck-Condon (FC) factors play a very important role for theoretical modeling and interpreting experimental observations for irradiative processes like electronic spectra of VUV absorption and fluorescence as well as the nonradiative processes like electron and energy transfer. ${ }^{\mathbf{1 - 1 0}}$ For large polyatomic molecules, multidimensional Franck-Condon overlapping integrals are conventionally decomposed into a product of one-dimensional Franck-Condon overlap integrals in terms of normal mode coordinates under harmonic oscillator approximation. Harmonic oscillator approximation provides the leading-term contribution to FC factors, and further higher order corrections of distorted oscillator approximation and mode-mixing including Duschinsky effect and anharmonic oscillators have been introduced to improve FC factors and its various applications. ${ }^{11-17}$ Most of the electronic molecular spectra and vibrational relaxation processes are experimentally

${ }^{a}$ Key Laboratory of Theoretical Chemistry of Environment, Ministry of Education, School of Chemistry \& Environment, South China Normal University, Guangzhou 510006, P. R. China. E-mail: gu@scnu.edu.cn

${ }^{b}$ Department of Applied Chemistry, Institute of Molecular Science and Center for Interdisciplinary Molecular Science, National Chiao-Tung University, Hsinchu 30050, Taiwan.E-mail: cyzhu@mail.nctu.edu.tw

$\dagger$ Electronic supplementary information (ESI) available. See DOI: 10.1039/c7ra00417f

$\ddagger$ Y. Hu and C.-W. Chen contributed equally to this work. carried out in solution phases. Therefore, theoretical modeling can deal with solutes and solvent molecules separately and FC factors including electronic and nuclear parts are treated separately as well. The electronic parts involve calculating electronic transition dipole momentum and nonadiabatic coupling matrix under solvent environment: the most popular theoretical approach is represented by continuum solvation models, especially the polarizable continuum model (PCM) combined with time-dependent density functional theory (TDDFT). The (TD)-DFT plus PCM approach provides an accurate simulation of static electronic potential energy surfaces of both ground and excited states for molecules in solution. The nuclear parts represent dynamic motion and its FC factors have been investigated based on various corrections to the leadingterm FC factors. ${ }^{18-36}$ By introducing damped harmonic oscillator approximation, Zhu and co-authors ${ }^{36}$ formulated the empirical correction to each normal mode differently in solvent environment by converting the mass-weighted unperturbed gasphase Hessian matrix to solution-phase perturbed Hessian matrix. This method is based on idea that each normal-mode motion can be affected differently in solution, however each local-mode motion can be affected in quite similar way for highly symmetric molecules like polycyclic aromatic hydrocarbon molecules in which $\mathrm{CH}$-stretch local modes, for example, are considered as in the same solvent environment. This method was previously demonstrated for interpreting solvent enhanced absorption and fluorescence spectra of 
perylene in benzene solution by applying a single empirical parameter to $\mathrm{CH}$-stretch local modes. ${ }^{36}$

One of the purposes in the present study is to continue damped harmonic oscillator FC simulation for much large size of polycyclic aromatic hydrocarbon like rubrene. The other is to interpret experimentally observed vibronic spectra in terms of potential energy surfaces of ground and excited states, transition patterns, molecular structure, solvent dependence and so on. For instance, it is traditionally considered that vibronic spectra simulated by FC factors are based on local minima between ground- and excited-state potential energy surfaces, and this is usually true for rigid molecules. However, for flexible molecules, potential energy surfaces are very complicated with many local minima closed to one another, in this case, (highorder) transition states that connect (more than) two local minima can play crucial role in FC simulation in order to interpret observed vibronic spectra.

Rubrene molecules have been attracted more attention in the field of organic semiconductor because of its high mobility at room temperature and optical properties, and rubrene can be used as a laser dye and a fluorescent dopant in organic light emitting devices, field effect transistors and solar cell. ${ }^{37-46}$ In the present study we focus on the optical properties of monomer of rubrene in non-polar benzene and polar cyclohexane solvents. Rubrene molecule is constructed with four six-member rings as the aromatic backbone connected by four phenyl rings perpendicular to the backbone. The backbone is the fluorescent center that can have planar or twisted geometry, and its optical spectra, however, are not well understood in terms of structure of the backbone geometry as well as phenyl groups. Moreover, it is well-know that rubrene has the large Stokes shift, but what is dependence in terms of solvent environment. We investigate how solvent enhanced absorption and fluorescence spectra vary with respect to solvents by damped FC simulation and identify physical origin of vibronic spectra in terms of ground- and excited-sate potential energy surfaces.

The rest of this paper is organized as follows: Section 2 briefly introduces damped harmonic oscillator FC factors and discuss (TD)-DFT+ PCM method for calculating potential energy surfaces of ground and excited states for rubrene molecule. Section 3 discusses how to reproduce experimentally observed absorption and fluorescence spectra in benzene and cyclohexane solution, and to interpret vibronic spectra in terms of molecular structure, molecular orbitals, vibrational normal modes and solvent environment. Section 4 presents concluding remarks.

\section{Damped Franck-Condon factors}

Within displaced harmonic oscillator approximation, absorption and fluorescence coefficients can be analytically derived as follows: $:^{1,42}$

$$
\begin{aligned}
\langle\alpha(\omega)\rangle & \propto \omega \int_{-\infty}^{\infty} \mathrm{d} t \mathrm{e}^{i t\left(\omega_{\mathrm{ba}}-\omega\right)-\frac{D_{\mathrm{ba}}{ }^{2} t^{2}}{4}-\gamma_{\mathrm{ba}}|t|} \\
& \times \exp \left[-\sum_{j} S_{j}\left\{\left(2 \bar{n}_{j}+1\right)-\left(\bar{n}_{j}+1\right) \mathrm{e}^{i t \omega_{j}}-\bar{n}_{j} \mathrm{e}^{-i t \omega_{j}}\right\}\right]
\end{aligned}
$$

and

$$
\begin{aligned}
\langle I(\omega)\rangle & \propto \omega^{3} \int_{-\infty}^{\infty} \mathrm{d} t \mathrm{e}^{-i t\left(\left|\omega_{\mathrm{ba}}\right|-\omega\right)-\frac{D_{\mathrm{ba}}{ }^{2} t^{2}}{4}-\gamma_{\mathrm{ba}}|t|} \\
& \times \exp \left[-\sum_{j} S_{j}\left\{\left(2 \bar{n}_{j}+1\right)-\left(\bar{n}_{j}+1\right) \mathrm{e}^{i t \omega_{j}}-\bar{n}_{j} \mathrm{e}^{-i t \omega_{j}}\right\}\right]
\end{aligned}
$$

where $\omega_{\text {ba }}$ represents electronically adiabatic excitation energy between electronic states $\mathrm{b}$ and a. $\bar{n}_{j}=\left(\mathrm{e}^{\hbar \omega_{j} / k_{\mathrm{B}} T}-1\right)^{-1}$ is the average phonon distribution, $\gamma_{\mathrm{ba}}$ is the homogeneous broadening parameter and $D_{\mathrm{ba}}$ is inhomogeneous broadening parameter that reflects static interaction between electronic states of molecule and solvent, and $\omega_{j}$ is the $j$-th normal mode vibrational frequency. The dimensionless Huang-Rhys factor $S_{j}$ corresponding to the $j$-th normal mode is defined as

$$
S_{j}=\frac{1}{2 \hbar} \omega_{j} d_{j}^{2}
$$

where displacement $d_{j}$ is given by

$$
d_{j}=Q_{j}^{\prime}-Q_{j}=\sum_{n} L_{j n}\left(q_{n}^{\prime}-q_{n}\right)
$$

The $q_{n}^{\prime}$ and $q_{n}$ in eqn (4) are the mass-weighted Cartesian coordinates at equilibrium geometries (or transition states) of the electronic excited and ground states, respectively. Transformation matrix $L$ in eqn (4) can be computed with frequency analysis using G09 programs, for instance. By applying damped diatomic harmonic oscillator to local-mode force constant of gas-phase Hessian matrix $H_{i j}$, we formulate damped FC factors as transferring the mass-weighted gas-phase unperturbed Hessian matrix to solvent perturbed Hessian matrix, ${ }^{\mathbf{3 6}}$

$$
H_{i \alpha, j \beta}^{0}=\frac{k_{i \alpha, j \beta}^{0}}{\sqrt{m_{i} m_{j}}} \rightarrow \frac{k_{i \alpha, j \beta}^{0}}{\sqrt{\left(m_{i} \zeta_{i}\right)\left(m_{j} \zeta_{j}\right)}}=\frac{k_{i \alpha, j \beta}}{\sqrt{m_{i} m_{j}}}=H_{i \alpha, j \beta},
$$

where $m_{i}$ and $m_{j}$ are atomic mass, and $\alpha$ and $\beta$ are Cartesian component $(x, y, z)$ of the position of nucleus $i$ and $j$. Unperturbed local force constant $k_{i \alpha, j \beta}^{0}$ is converted to perturbed force constant $k_{i \alpha, j \beta}=k_{i \alpha, j \beta}^{0} / \sqrt{\zeta_{i} \zeta_{j}}$. The dimensionless scaling parameter $\zeta_{j}$ in eqn (5) is treated as an empirical parameter that can be considered as a local dynamic interaction of atom $j$ of solute molecule with solvent molecules. By diagonalizing perturbed Hessian matrix in eqn (5), we obtain normal mode frequencies and transformation matrix (that transfers the massweighted Cartesian coordinates to normal mode coordinates) corresponding to solute molecule under the solvent environment. It should be emphasized that solvent effect by damped oscillator can directly modify the leading-term of FC factors, so that it is usually larger than the other effects such as distorted oscillator, Duschinsky effect and anharmonic effect which are in higher-order perturbation terms.

We have employed the (time-dependent) density functional theory with functional (TD)-B3LYP ${ }^{47}$ (i.e., B3LYP20), (TD) B3LYP35, (TD)-B3LYP50 (i.e., BHandHLYP), ${ }^{48}$ and HF-CIS (i.e., B3LYP100) plus PCM condition in benzene and cyclohexane solvents for calculating potential energy surfaces of ground and the first excited states. The numbers following after B3LYP 
stand for different hybrid exchange-correlation functionals containing $20 \%$ (B3LYP), 35\%, 50\%, and $100 \%$ of exact HartreeFock exchange in the density functional theory. The basis set is chosen as 6-31G throughout all calculations and all calculations are carried out using GAUSSIAN 09 program package. ${ }^{49} \mathrm{Calcu}-$ lations from four functionals given above lead to the same conclusion in which there are two local minima with $D_{2}$, two local minima with $C_{2 \mathrm{H}}$, and one second-order transition state with $D_{2 \mathrm{H}}$ group symmetry in both ground- and excited-state potential energy surfaces. This conclusion does hold for PCM condition in both non-polar benzene and polar cyclohexane solvents although electronic structures can vary slightly. From preliminary vibronic spectral calculation, we found that (TD)B3LYP50 presents the best agreement with experimental results among four functionals, and this is the same as results obtained in ref. 42. However, there is important difference in comparison with ref. 42 in which there are only two local minima, one is in $D_{2}$ and the other is in $D_{2 \mathrm{H}}$ symmetry (note for this point, we show it is second-order transition state). The Huang-Rhys factors calculated from our $D_{2 \mathrm{H}}$ structure are similar to those from their $D_{2 \mathrm{H}}$ symmetry, but the Huang-Rhys factors calculated from our $D_{2}$ structure are very different to those from their $D_{2}$ symmetry. Actually, the second-order transition point at $D_{2 \mathrm{H}}$ connects two local minima at $D_{2}$ as well as two local minima at $C_{2 \mathrm{H}}$, and we will discuss this in detail later. In the following discussions, we only report calculations from (TD)-BHandHLYP (B3LYP50) as it performs best for vibronic spectral simulations.

\section{Results and analysis}

Rubrene molecule plays as a prototype of large-size polycyclic aromatic hydrocarbon molecules for a better understanding of molecular structure, spectra, dynamics, solvent effect, and so on. It's highly symmetrical electronic structures (see atomic numbering in Fig. 1) in which all hydrogen atoms are approximately subjected to the same environment interacting with solvent molecules and this greatly simplifies empirical search of the scaling parameters in damped FC simulation. Geometry of rubrene molecule is composed with four linearly connected sixmember rings as aromatic backbone connected by four phenyl rings perpendicular to the backbone. The present (TD)-DFT calculations reveal that there are two local minima at $C_{2 \mathrm{H}}$ group symmetry where $C_{2 \mathrm{H}}(1)$ minimum is mirror image of $C_{2 \mathrm{H}}(2)$ minimum as shown in Fig. 2(a) and (b), there is one second-order transition state at $D_{2 \mathrm{H}}$ group symmetry as shown in Fig. 2(c), and there are two local minima at $D_{2}$ group symmetry where $D_{2}(1)$ minimum is mirror image of $D_{2}(2)$ minimum as shown in Fig. 2(d) and (e). These kind of electronic conformers displayed in Fig. 2 show similar structures for ground and excited states in both benzene and cyclohexane solvents. At $D_{2 \mathrm{H}}$ transition state, there are actually two normal modes with imaginary frequencies $-58.62 \mathrm{~cm}^{-1}$ and -54.49 $\mathrm{cm}^{-1}\left(-58.06 \mathrm{~cm}^{-1}\right.$ and $\left.-53.21 \mathrm{~cm}^{-1}\right)$ in Au and B3G symmetries, respectively for the ground (excited) state in benzene solvent. Along Au and B3G normal-mode coordinates, we draw two-dimensional potential energy surface diagrams in which

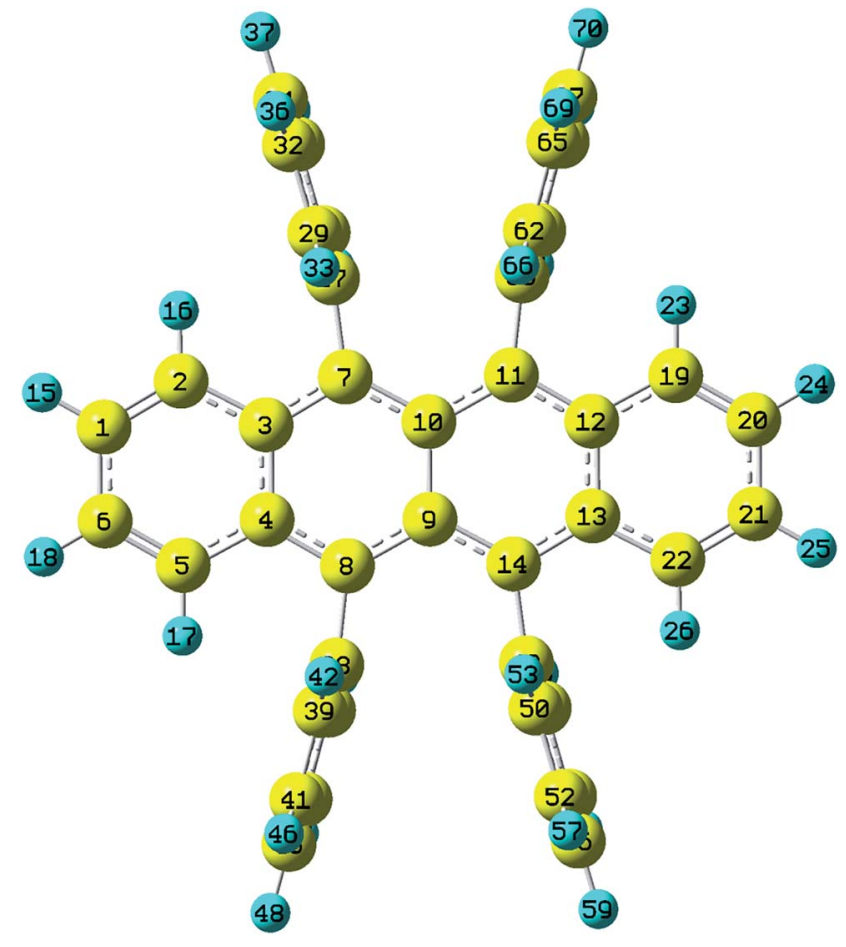

Fig. 1 Atomic numbering (optimized geometries under $D_{2 H}$ group symmetry by BHandHLYP/6-31G plus PCM model. The yellow and blue indicate carbon and hydrogen atoms respectively).

second-order $D_{2 \mathrm{H}}$ transition state connects two $D_{2}$ minima via $\mathrm{Au}$ and two $C_{2 \mathrm{H}}$ minima via $\mathrm{B} 3 \mathrm{G}$ as shown in Fig. 3(a) for ground state and Fig. 3(b) for excited state in benzene solvent environment. Similar potential energy surface diagrams in cyclohexane solvent environment are also shown in Fig. 4(a) and (b), where two imaginary frequencies are $-56.54 \mathrm{~cm}^{-1}$ and -52.85 $\mathrm{cm}^{-1}\left(-55.70 \mathrm{~cm}^{-1}\right.$ and $\left.-51.29 \mathrm{~cm}^{-1}\right)$ with $\mathrm{Au}$ and B3G mode symmetries, respectively for the ground (excited) state. Difference between ground and excited state potential energy surfaces is not large, and this confirms that the present displaced harmonic FC simulation should be good approximation to reproduce experimental vibronic spectra. The adiabatic potential energy gap between $D_{2 \mathrm{H}}$-transition state and $D_{2}$-local minima $\left(C_{2 \mathrm{H}^{-}}\right.$local minima) is $0.31 \mathrm{eV}(0.12 \mathrm{eV})$ as shown in Fig. 3(a) and 4(a) for $\mathrm{S}_{0}$ state and is $0.51 \mathrm{eV}(0.20 \mathrm{eV})$ as shown in Fig. 3(b) and 4(b) for $\mathrm{S}_{1}$ state, respectively in both benzene and cyclohexane solvents. Potential energy surfaces around $D_{2 \mathrm{H}^{-}}$ transition state in terms of two imaginary normal-mode coordinates show little dependence on solvent.

\subsection{Electronic structures, potential energy surfaces and the Huang-Rhys factors}

The present (TD)-DFT+ PCM calculations reveal that the backbone formed by the four six-member rings is planar structure at local minima $C_{2 \mathrm{H}}(1), C_{2 \mathrm{H}}(2)$ and the second-order transition state $D_{2 \mathrm{H}}$, but the backbone is twisted at local minima $D_{2}(1)$ and $D_{2}(2)$ for both $\mathrm{S}_{0}$ and $\mathrm{S}_{1}$ states. Since there are existing $D_{2}, C_{2 \mathrm{H}}$ and $D_{2 \mathrm{H}}$ group symmetries at five optimized geometries for both 


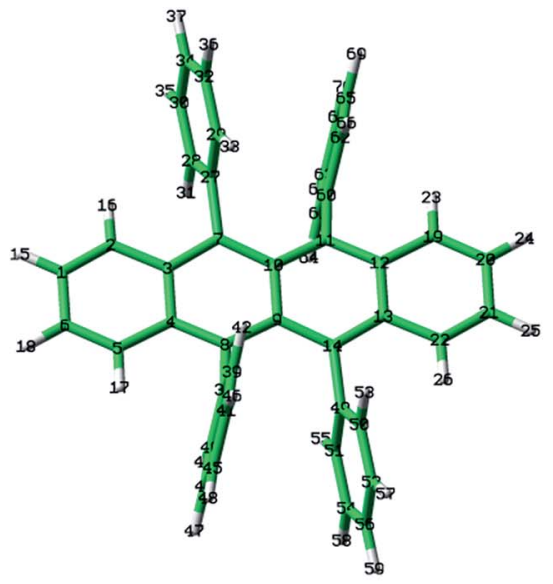

(a)

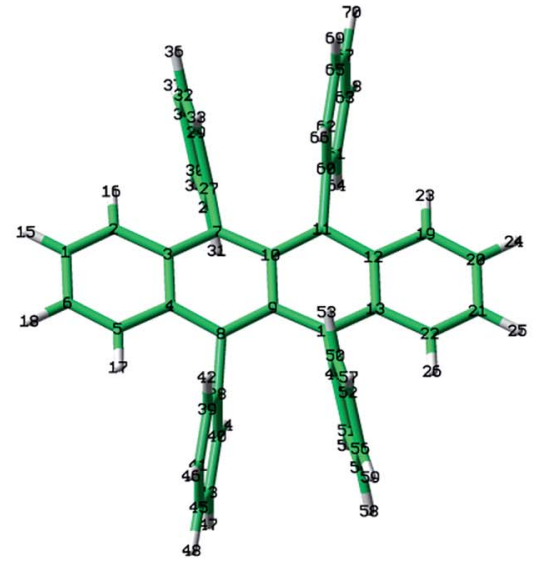

(b)

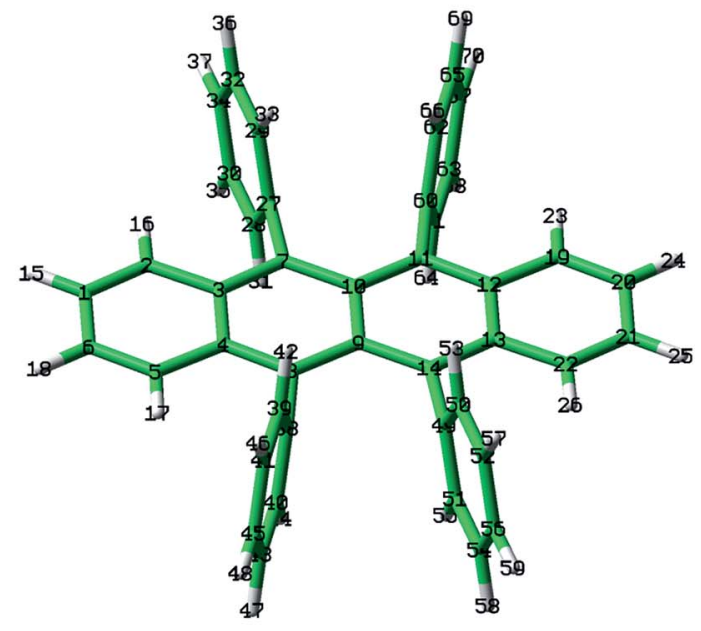

(c)

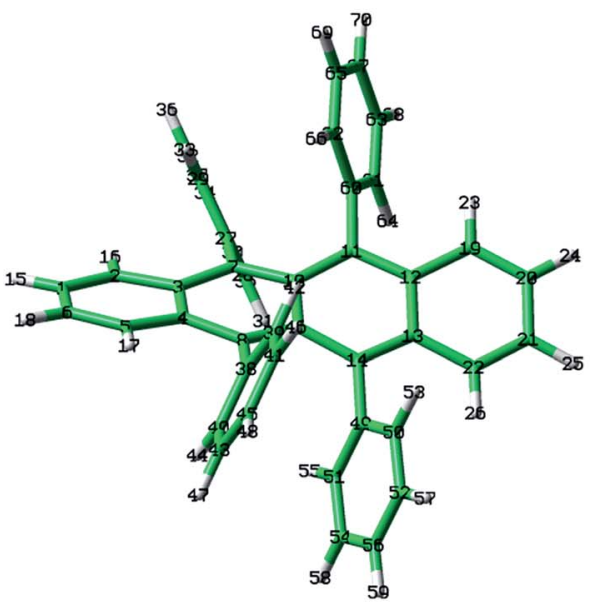

(d)

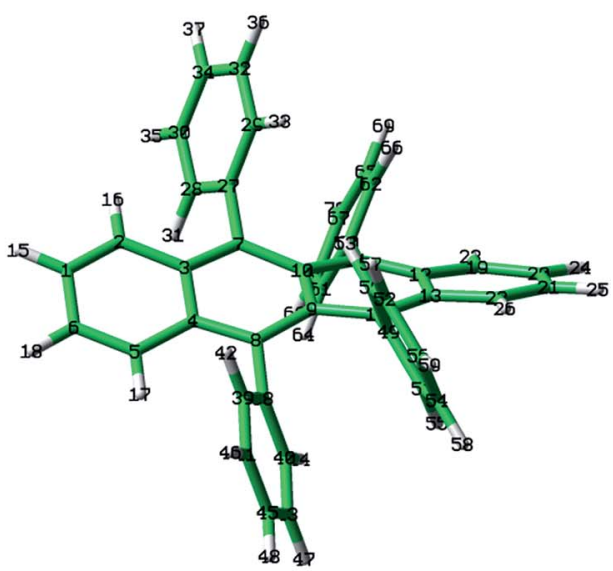

(e)

Fig. 2 Optimized geometries by BHandHLYP/6-31G plus PCM model in benzene solvent for ground-state $S_{0}$. Local minima (a) $C_{2 H}(1)$ and (b) $C_{2 H}(2)$, (c) second-order transition state $D_{2 H}$, and local minima (d) $D_{2}(1)$ and (e) $D_{2}(2)$.

$\mathrm{S}_{0}$ and $\mathrm{S}_{1}$ states, the internal coordinates of selected bond lengths, bond angles and dihedral angles which well represent geometries of electronic structures contributing to vibronic spectra are given in Tables 1 and 2 . Tables 1 and 2 actually show difference of bond lengths, bond angles and dihedral angles between $S_{0}$ and $S_{1}$ states with the same group symmetry in 


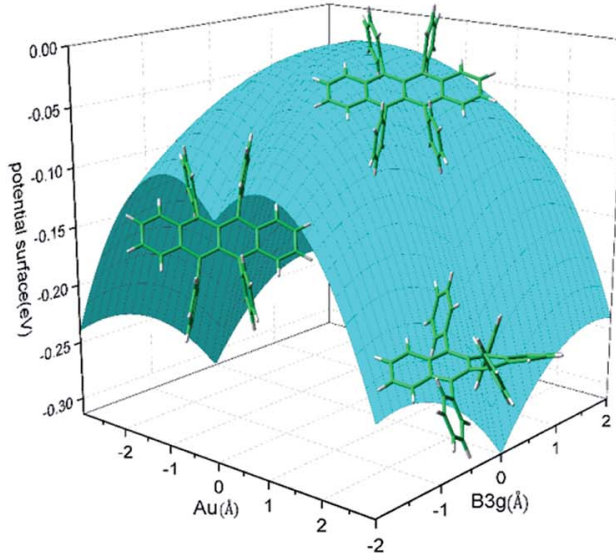

(a)

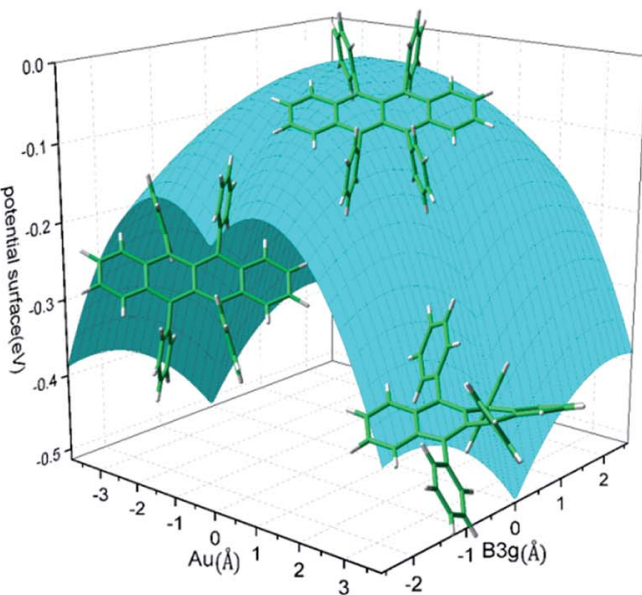

(b)

Fig. 3 Potential energy surface along two imaginary normal-mode coordinates (Au and B3G symmetries) starting from $D_{2 H}$ transition state point which connects two $C_{2 H}$ minima via B3G-mode direction, and two $D_{2}$ minima via Au-mode direction. (a) Ground state and (b) excited state in benzene solvent

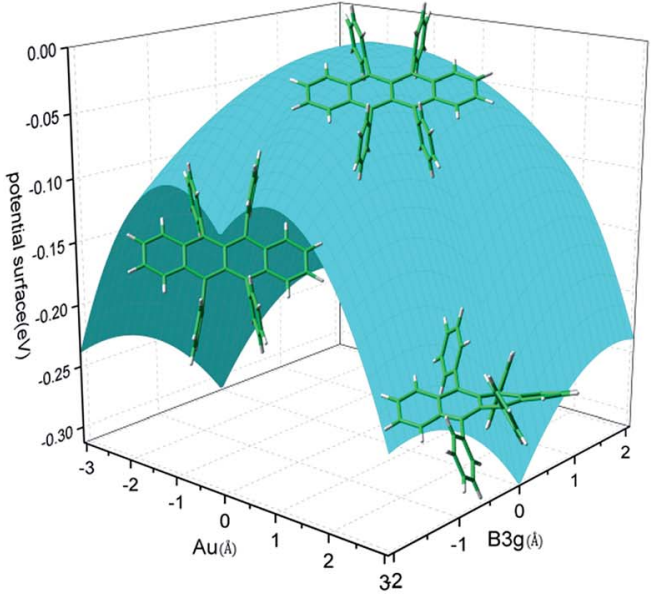

(a)

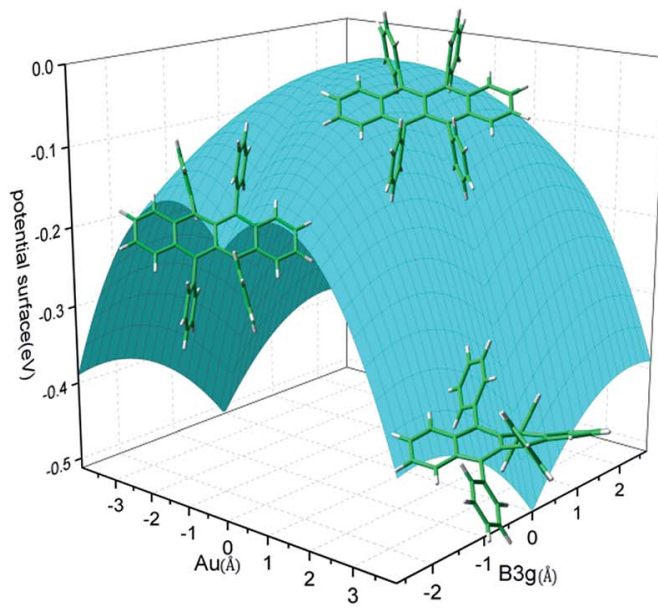

(b)

Fig. 4 Potential energy surface along two imaginary normal-mode coordinates (Au and B3G symmetries) starting from $D_{2 H}$ transition state point which connects two $C_{2 H}$ minima via B3G-mode direction, and two $D_{2}$ minima via Au-mode direction. (a) Ground state and (b) excited state in cyclohexane solvent.

benzene and cyclohexane solvents, respectively, and the corresponding absolute values of those internal coordinates are shown in Tables S1 and $\mathrm{S} 2 \dagger$ (Full Cartesian coordinates for optimized geometries shown in Table S3) (ESI $\dagger$ ). As is wellknown, the magnitude of Huang-Rhys factor defined in eqn (3) depends on the displacement of the normal mode between local minimum on ground state and corresponding local minimum on excited state, while the displacement of normal mode is converted from differences of internal coordinates between local minima of the ground and excited states. Bondlength differences vary with respect change of group symmetry from $D_{2 \mathrm{H}}$ to $C_{2 \mathrm{H}}$ and from $D_{2 \mathrm{H}}$ to $D_{2}$ within small magnitude (the largest value is about $0.05 \AA$ for $\mathrm{C} 9 \mathrm{C} 10$ bond in benzene solvent) as shown in Tables 1 and 2, and this can lead the change of Huang-Rhys factor about 0.4. However, bondangle differences can vary within as large as $6^{\circ}$, and this can lead to the change of Huang-Rhys factor about 4 . The largest change happens for dihedral angles as shown in Tables 1 and 2 and it can go as large as ten degree, but these changes affect change of Huang-Rhys factor in complicated way and it is hard to predict the magnitude change before carrying out real calculations. This fact was actually confirmed in which the Huang-Rhys factors of the some vibrational modes can go as large as from 5 to 50 due to big change in dihedral angles for the two isomeric compounds. ${ }^{50}$ Dihedral angle differences between $\mathrm{S}_{0}$ and $\mathrm{S}_{1}$ states at $D_{2 \mathrm{H}}$ group symmetry are all equal to zero as 
Table 1 Differences of bond lengths (in $\AA$ ), bond angles and dihedral angles (in degree) between ground and excited states and numbers of $S_{1}$ minus $S_{0}$ are listed for optimized representative geometries within $D_{2}, D_{2 H}$, and $C_{2 H}$ group symmetries and all are computed by (TD)BHandHLYP/6-31G plus PCM model in benzene solvent (the atom numbering is showed in Fig. 1)

\begin{tabular}{|c|c|c|c|c|c|}
\hline \multirow[b]{2}{*}{ Parameters } & \multicolumn{5}{|c|}{ Conformations } \\
\hline & $D_{2}(1)$ & $D_{2}(2)$ & $D_{2 \mathrm{H}}$ & $C_{2 \mathrm{H}}(1)$ & $C_{2 \mathrm{H}}(2)$ \\
\hline$B(1,2)$ & 0.0285 & 0.0285 & 0.0304 & 0.0288 & 0.0288 \\
\hline$B(1,6)$ & -0.0315 & 0.0315 & -0.0319 & -0.0307 & -0.0307 \\
\hline$B(1,15)$ & -0.0004 & 0.0004 & -0.0002 & 0.0001 & 0.0001 \\
\hline$B(2,3)$ & -0.0304 & 0.0304 & -0.0309 & -0.2976 & -0.2976 \\
\hline$B(2,16)$ & 0.0000 & 0.0000 & 0.0001 & 0.0001 & 0.0001 \\
\hline$B(3,4)$ & -0.0072 & 0.0072 & -0.0086 & -0.0069 & -0.0069 \\
\hline$B(3.7)$ & 0.0387 & -0.0387 & 0.0346 & 0.0367 & 0.0367 \\
\hline$B(7,10)$ & -0.0173 & 0.0173 & -0.0098 & -0.0095 & -0.0095 \\
\hline$B(7,27)$ & -0.0084 & 0.0084 & -0.0037 & -0.0076 & -0.0076 \\
\hline$B(8,38)$ & -0.0084 & 0.0084 & -0.0043 & -0.0076 & -0.0076 \\
\hline$B(9,10)$ & 0.0638 & -0.0638 & 0.0134 & 0.0009 & 0.0009 \\
\hline$B(39,42)$ & -0.0002 & 0.0002 & -0.0001 & -0.0003 & -0.0003 \\
\hline$A(1,2,3)$ & 0.1300 & -0.1300 & -0.0100 & -0.0200 & -0.0200 \\
\hline$A(2,3,4)$ & 0.4000 & -0.4000 & 0.4300 & 0.4000 & 0.4000 \\
\hline$A(2,1,6)$ & -0.4200 & 0.4200 & -0.3600 & -0.3900 & -0.3900 \\
\hline$A(3,7,10)$ & 0.2500 & -0.2500 & 0.8600 & 0.3000 & 0.3000 \\
\hline$A(4,3,7)$ & -0.5600 & 0.5600 & -0.7000 & -0.9400 & -0.9400 \\
\hline$A(7,10,9)$ & -0.4000 & 0.4000 & -0.2200 & -0.0500 & -0.0500 \\
\hline$D(3,7,27,29)$ & 8.1500 & -8.1500 & 0.0000 & 9.4600 & -7.9000 \\
\hline$D(4,8,38,39)$ & 6.1400 & -6.1400 & 0.0000 & -9.4600 & 7.9000 \\
\hline$D(2,3,4,8)$ & -1.3900 & 1.3900 & 0.0000 & -0.0200 & 0.0200 \\
\hline$D(3,7,10,11)$ & -3.9100 & 3.9100 & 0.0000 & -4.2600 & 4.2600 \\
\hline$D(1,2,3,4)$ & 1.0300 & -1.0300 & 0.0000 & -0.4600 & 0.4600 \\
\hline$D(7,10,9,8)$ & 4.7800 & -4.7800 & 0.0000 & 0.0000 & 0.0000 \\
\hline
\end{tabular}

shown in the middle column of Tables 1 and 2, and thus this indicates that observed vibronic spectra should be mostly affected by the planar backbone structure. We will confirm this issue later.

Experimentally observed absorption and fluorescence spectra in benzene and cyclohexane solvents are mostly from the lowest singlet excited state $S_{1}$ of rubrene molecule with transition type of molecular orbital $\pi \rightarrow \pi^{*}$. The present calculations show that the first excited state $S_{1}$ has $\pi \rightarrow \pi^{*}$ feature, and natural orbital analysis confirm that the $S_{1}$ state results mainly in the excitation of the HOMO $\rightarrow$ LUMO (one electron excited) as shown in Fig. S1 (ESI $\dagger$ ) corresponding to $\mathrm{S}_{0}$ $\left(\mathrm{X}^{1} \mathrm{~A}_{\mathrm{g}}\right) \rightarrow \mathrm{S}_{1}\left(\mathrm{~A}^{1} \mathrm{~B}_{3 \mathrm{u}}\right)$ electronic excitation between $\mathrm{S}_{0}-D_{2 \mathrm{H}}$ and $\mathrm{S}_{1}-D_{2 \mathrm{H}}$ transition states. Fig. S1 (ESI $\dagger$ ) show that electron density is located on the backbone in both HOMO and LUMO, so that four phenyl groups perpendicular to the backbone are not active for vibronic spectra. Therefore, this indicates that absorption and fluorescence spectra are mostly influenced by electronic transition associate with the backbone of rubrene, and solvent enhanced vibronic spectra should be also focused on carbon and hydrogen atoms in the backbone. Actually, the present (TD)-DFT calculations shows that adiabatic (vertical) excitation energies are $2.506 \mathrm{eV}$ and $2.514 \mathrm{eV}(2.753 \mathrm{eV}$ and $2.760 \mathrm{eV})$ in benzene and cyclohexane solvents, respectively. Due to the backbone of rubrene is rigid frame, polar and non-polar solvents do not affect electronic excitation and even oscillator
Table 2 Differences of bond lengths (in $\AA$ ), bond angles and dihedral angles (in degree) between ground and excited states and numbers of $S_{1}$ minus $S_{0}$ are listed for optimized representative geometries within $D_{2}, D_{2 H}$ and $C_{2 H}$ group symmetries and all are computed by (TD)BHandHLYP/6-31G plus PCM model in cyclohexane solvent (the atom numbering is showed in Fig. 1)

\begin{tabular}{|c|c|c|c|c|c|}
\hline \multirow[b]{2}{*}{ Parameters } & \multicolumn{5}{|c|}{ Conformations } \\
\hline & $D_{2}(1)$ & $D_{2}(2)$ & $D_{2 \mathrm{H}}$ & $C_{2 \mathrm{H}}(1)$ & $C_{2 \mathrm{H}}(2)$ \\
\hline$B(1,2)$ & 0.0286 & 0.0286 & 0.0404 & 0.0289 & 0.0289 \\
\hline$B(1,6)$ & -0.0314 & -0.0314 & -0.0319 & -0.0306 & -0.0306 \\
\hline$B(1,15)$ & -0.0003 & -0.0003 & -0.0002 & -0.0002 & -0.0002 \\
\hline$B(2,3)$ & -0.0305 & -0.0305 & -0.0273 & -0.0306 & -0.0306 \\
\hline$B(2,16)$ & 0.0000 & 0.0000 & 0.0002 & 0.0002 & 0.0002 \\
\hline$B(3,4)$ & -0.0071 & -0.0071 & -0.0086 & -0.0068 & -0.0068 \\
\hline$B(3.7)$ & 0.0387 & 0.0387 & 0.0346 & 0.0365 & 0.0365 \\
\hline$B(7,10)$ & -0.0144 & -0.0144 & -0.0097 & -0.0095 & -0.0095 \\
\hline$B(7,27)$ & -0.0084 & -0.0084 & -0.0038 & -0.0075 & -0.0075 \\
\hline$B(8,38)$ & -0.0084 & -0.0084 & -0.0038 & -0.0075 & -0.0075 \\
\hline$B(9,10)$ & 0.0168 & 0.0168 & 0.0134 & 0.0010 & 0.0010 \\
\hline$B(39,42)$ & -0.0002 & -0.0002 & 0.0000 & -0.0003 & -0.0003 \\
\hline$A(1,2,3)$ & 0.1300 & 0.1300 & -0.0100 & -0.0200 & -0.0200 \\
\hline$A(2,3,4)$ & 0.3900 & 0.3900 & 0.4300 & 0.4000 & 0.4000 \\
\hline$A(2,1,6)$ & -0.9700 & -0.9700 & -0.4200 & -0.3900 & -0.3900 \\
\hline$A(3,7,10)$ & 0.2600 & 0.2600 & 0.8600 & 0.3200 & 0.3200 \\
\hline$A(4,3,7)$ & -0.5700 & -0.5700 & -0.7000 & -0.9300 & -0.9300 \\
\hline$A(7,10,9)$ & -0.4100 & -0.4100 & -0.1800 & 0.0500 & 0.0500 \\
\hline$D(3,7,27,29)$ & 8.1600 & -8.1600 & 0.0000 & 9.2900 & -7.7100 \\
\hline$D(4,8,38,39)$ & 6.1500 & -6.1500 & 0.0000 & -9.2900 & 7.7100 \\
\hline$D(2,3,4,8)$ & -1.3600 & 1.3600 & 0.0000 & -0.0200 & 0.0200 \\
\hline$D(3,7,10,11)$ & -3.9000 & 3.9000 & 0.0000 & -4.2200 & 4.2200 \\
\hline$D(1,2,3,4)$ & 0.9900 & -0.9900 & 0.0000 & -0.4600 & 0.4600 \\
\hline$D(7,10,9,8)$ & 4.7800 & -4.7800 & 0.0000 & 0.0000 & 0.0000 \\
\hline
\end{tabular}

strengths are about the same as 0.33 for $\mathrm{S}_{0}\left(\mathrm{X}^{1} \mathrm{~A}_{\mathrm{g}}\right) \rightarrow \mathrm{S}_{1}\left(\mathrm{~A}^{1} \mathrm{~B}_{3 \mathrm{u}}\right)$ excitation. The present (TD)-DFT calculations show good agreement with experimental data and the other theoretical calculations as shown in Table 3.

Franck-Condon factors can be qualitatively estimated from Huang-Rhys factors $S$ (that are also called as vibronic coupling); when $S$ is smaller than 1, peak position of vibronic spectra is close to adiabatic excitation energy $\omega_{\text {ba }}$ (or $0-0$ vibronic transition energy), and when $S$ is larger than 1 , the peak position is far from $\omega_{\mathrm{ba}}$, and the larger $S$ is, the farther from $\omega_{\mathrm{ba}}$. Therefore, Huang-Rhys factors can present an immediate comparison with experimentally observed spectra to determine which electronic structure is an actually contributed observed spectrum. Let us calculate Huang-Rhys factors based on $\mathrm{S}_{0^{-}} D_{2}(1)$ and $\mathrm{S}_{1^{-}}$ $D_{2}(1)$ local minima (it is the same based on $\mathrm{S}_{0}{ }^{-} D_{2}(2)$ and $\mathrm{S}_{1}$ $D_{2}(2)$ ), then we found there are $S=15$ and $S=13$ that correspond to vibrational frequencies $1565 \mathrm{~cm}^{-1}$ and $3331 \mathrm{~cm}^{-1}$, respectively in both benzene and cyclohexane solvents and its vibrational normal-mode motions are related to large change of dihedral angles in phenyl rings as shown in Fig. S2 (ESI†). Such large Huang-Rhys factors present vibronic spectra totally wrong in comparison with experimental results and actually peak positions of vibronic spectra in experiment tell that all $S$ values should be smaller than unity. The same is also true if we analyze calculated Huang-Rhys factors based on $\mathrm{S}_{0}-C_{2 \mathrm{H}}(1)$ and $\mathrm{S}_{1}-C_{2 \mathrm{H}}(1)$ local minima, because there are $S=23$ and $S=13$ that 
Table 3 Observed and calculated vertical and adiabatic excitation energies $\left(E_{\mathrm{vert}}(\mathrm{eV})\right.$ and $\left.E_{\mathrm{ad}}(\mathrm{eV})\right)$ with corresponding oscillator strengths $(f)$ for ${ }^{1} \mathrm{~B}_{3 u}$ transition and discrepancies $(\Delta)$ between adiabatic excitation energies from theory and experiment

\begin{tabular}{|c|c|c|c|c|}
\hline \multirow[b]{2}{*}{ Solvent } & \multicolumn{2}{|c|}{ Vertical } & \multicolumn{2}{|c|}{ Adiabatic } \\
\hline & $E_{\text {vert }}$ & $f$ & $E_{\text {ad }}(\mathrm{eV})$ & $\Delta(\mathrm{eV})$ \\
\hline \multirow{4}{*}{$\begin{array}{l}\text { Benzene } \\
\text { Cyclohexane }\end{array}$} & 2.753 & 0.338 & 2.506 & \\
\hline & $2.487^{a}$ & $0.13^{a}$ & $2.302^{a}$ & \\
\hline & 2.760 & 0.328 & 2.514 & 0.21 \\
\hline & $2.795^{b}$ & $0.32^{b}$ & $2.599^{b}$ & 0.29 \\
\hline
\end{tabular}

${ }^{a}$ Experiment values from ref. $42 .{ }^{b}$ Calculated values from ref. 42.

correspond to vibrational frequencies $1563 \mathrm{~cm}^{-1}$ and 3330 $\mathrm{cm}^{-1}$ respectively, and its normal-mode motions are as shown in Fig. S3(ESI $\dagger$ ). We performed fluorescence spectral calculations based on both $D_{2}$ and $C_{2 \mathrm{H}}$ symmetries and that HuangRhys factors computed from $\mathrm{S}_{1}-D_{2}$ and $\mathrm{S}_{1}-C_{2 \mathrm{H}}$ local minima also show very large $S$ values as discussed as above for absorption spectrum. Thus, we conclude that observed vibronic spectra cannot be interpreted by molecular structures related to $D_{2}$ and $C_{2 \mathrm{H}}$ group symmetries.

Only when we calculate Huang-Rhys factors based on the second-order transition states of $S_{0}-D_{2 H}$ and $S_{1}-D_{2 H}$, we have all $S$ values are smaller than unity as shown in Tables 4 and 6, and thus it is only $D_{2 \mathrm{H}}$ group symmetry that can interpret experimentally observed vibronic spectra. In the following subsections, we will discuss Franck-Condon simulation based on only the second-order transition states of $D_{2 \mathrm{H}}$ group symmetry. By performing frequency calculation at geometry of secondorder transition state $S_{0}\left(S_{1}\right)$ in benzene solvent environment, we found 23 (21) total symmetry $\mathrm{A}_{\mathrm{g}}$ vibrational modes with nonzero $S$ values, while in cyclohexane solvent environment, we found 20 (22) out of 208 vibrational normal modes. Number of $\mathrm{A}_{\mathrm{g}}$ modes with non-zero $S$ values is slightly different in benzene and cyclohexane solvents, however, this slight difference does not affect vibronic spectra so much. For absorption spectra, the Huang-Rhys factors are calculated based on frequency and its transformation matrix at $\mathrm{S}_{0}-D_{2 \mathrm{H}}$, there are four active normal modes $\left(v_{15}, v_{16}, v_{17}\right.$, and $\left.v_{20}\right)=(1389,1416,1555$, and 1669) $\mathrm{cm}^{-1}$ corresponding to $S$-value $0.356,0.05,0.037$, and 0.137 , respectively within benzene solvent and $\left(v_{13}, v_{14}, v_{15}\right.$, and $\left.v_{17}\right)=$ $(1390,1415,1555$, and 1669$) \mathrm{cm}^{-1}$ corresponding to $S$-value $0.356,0.05,0.037$, and 0.137 , respectively within cyclohexane solvent. All Huang-Rhys factors for $\mathrm{A}_{\mathrm{g}}$ modes are listed in no scaling $\left(\zeta_{\mathrm{H}}=1.0\right)$ column of Table 4 for benzene solvent and in Table 6 for cyclohexane solvent, four active normal modes all vibrates as ring wagging plus $\mathrm{CC}$ stretch and $\mathrm{CH}$ bend motion on the backbone as shown in Fig. S4(a) and S5(a) (ESI $\dagger$ ). We can immediately conclude that absorption spectrum should be the same in non-polar benzene and polar cyclohexane solvents in which the four active normal-mode motions are almost the exactly same. For fluorescence spectra, the Huang-Rhys factors are calculated based on frequency and its transformation matrix

Table 4 Calculated vibrational normal-mode frequencies $\omega$ (in $\mathrm{cm}^{-1}$ ) and corresponding Huang-Rhys factors $S$ (only for non-zero values) vary with respect to the scaling parameters for absorption spectrum in benzene solvent. The scaling parameters $\zeta$ for 8 backbone- $\mathrm{H}$ atoms $15,16,17$, $18,23,24,25$, and 26 (see Fig. 1) are changed equally as listed below and for the rest of hydrogen atoms and all carbon atoms are fixed as $\zeta_{H}=1.0$ and $\zeta_{C}=1.0$ (no scaling)

\begin{tabular}{|c|c|c|c|c|c|c|c|c|c|}
\hline & & \multicolumn{2}{|c|}{$\underline{\zeta_{\mathrm{H}}}=1.0$} & \multicolumn{2}{|c|}{$\zeta_{\mathrm{H}}=1.4$} & \multicolumn{2}{|c|}{$\zeta_{\mathrm{H}}=1.6$} & \multicolumn{2}{|c|}{$\zeta_{\mathrm{H}}=2.0$} \\
\hline & & $\omega$ & $S$ & $\omega$ & $S$ & $\omega$ & $S$ & $\omega$ & $S$ \\
\hline 2 & & 213 & 0.021 & 210 & 0.019 & 208 & 0.019 & 205 & 0.019 \\
\hline 3 & & 274 & 0.035 & 267 & 0.037 & 263 & 0.033 & 256 & 0.032 \\
\hline 4 & & 357 & 0.096 & 350 & 0.094 & 346 & 0.095 & 339 & 0.100 \\
\hline 7 & & 706 & 0.020 & 685 & 0.003 & 661 & 0.019 & 650 & 0.022 \\
\hline 8 & & 772 & 0.004 & 695 & 0.019 & 688 & 0.018 & 675 & 0.015 \\
\hline 9 & & 829 & 0.004 & 777 & 0.001 & 754 & 0.001 & 712 & 0.000 \\
\hline 10 & & 1043 & 0.022 & 985 & 0.000 & 955 & 0.000 & 902 & 0.003 \\
\hline 11 & & 1078 & 0.004 & 1019 & 0.020 & 978 & 0.002 & 918 & 0.016 \\
\hline 12 & & 1267 & 0.018 & 1104 & 0.009 & 1041 & 0.001 & 1015 & 0.025 \\
\hline 17 & & 1555 & 0.037 & 1484 & 0.146 & 1467 & 0.222 & 1449 & 0.288 \\
\hline 18 & & 1611 & 0.005 & 1527 & 0.001 & 1506 & 0.001 & 1480 & 0.000 \\
\hline 19 & & 1623 & 0.014 & 1590 & 0.001 & 1588 & 0.001 & 1585 & 0.000 \\
\hline 20 & & 1669 & 0.137 & 1656 & 0.237 & 1653 & 0.231 & 1646 & 0.247 \\
\hline 21 & & 3320 & 0.004 & 2855 & 0.001 & 2695 & 0.001 & 2455 & 0.001 \\
\hline 22 & & 3329 & 0.034 & 2863 & 0.025 & 2702 & 0.016 & 2461 & 0.006 \\
\hline 23 & & 3396 & 0.007 & 2920 & 0.005 & 2757 & 0.002 & 2512 & 0.001 \\
\hline
\end{tabular}


Table 5 Calculated vibrational normal-mode frequencies $\omega$ (in $\mathrm{cm}^{-1}$ ) and corresponding Huang-Rhys factors $S$ (only for non-zero values) vary with respect to the scaling parameters $\zeta$ for fluorescence spectra in benzene solvent. The scaling parameters $\zeta$ for 8 backbone- $\mathrm{H}$ atoms plus 12 backbone-C atoms 1, 2, 3, 4, 5, and 6, and 12, 13, 19, 20, 21, and 22 (see Fig. 1) are varied as listed below and for the rest of hydrogen atoms and the rest of carbon atoms are fixed as $\zeta_{H}=1.0$ and $\zeta_{\mathrm{C}}=1.0$ (no scaling)

\begin{tabular}{|c|c|c|c|c|c|c|c|c|}
\hline \multirow{2}{*}{$\begin{array}{l}\text { Total symmetry vibration } \\
\text { modes }\left(A_{g}\right)\end{array}$} & \multicolumn{2}{|c|}{$\zeta_{\mathrm{H}}=1.0, \zeta_{\mathrm{C}}=1.0$} & \multicolumn{2}{|c|}{$\zeta_{\mathrm{H}}=1.6, \zeta_{\mathrm{C}}=1.2$} & \multicolumn{2}{|c|}{$\zeta_{\mathrm{H}}=1.8, \zeta_{\mathrm{C}}=1.2$} & \multicolumn{2}{|c|}{$\zeta_{\mathrm{H}}=2.3, \zeta_{\mathrm{C}}=1.2$} \\
\hline & $\omega$ & $S$ & $\omega$ & $S$ & $\omega$ & $S$ & $\omega$ & $S$ \\
\hline 1 & 93 & 0.0141 & 93 & 0.0141 & 93 & 0.0141 & 93 & 0.0141 \\
\hline 2 & 210 & 0.0224 & 210 & 0.0225 & 210 & 0.0225 & 210 & 0.0225 \\
\hline 3 & 272 & 0.0356 & 264 & 0.0537 & 264 & 0.0536 & 262 & 0.0582 \\
\hline 4 & 354 & 0.0984 & 332 & 0.0520 & 331 & 0.0475 & 328 & 0.0428 \\
\hline 5 & 583 & 0.0115 & 580 & 0.0110 & 580 & 0.0091 & 579 & 0.0093 \\
\hline 6 & 670 & 0.0139 & 620 & 0.0192 & 616 & 0.0151 & 606 & 0.0119 \\
\hline 7 & 706 & 0.0142 & 690 & 0.0044 & 689 & 0.0023 & 688 & 0.0021 \\
\hline 8 & 828 & 0.0062 & 772 & 0.0056 & 772 & 0.0053 & 772 & 0.0034 \\
\hline 9 & 1059 & 0.0127 & 971 & 0.0054 & 943 & 0.0122 & 926 & 0.0004 \\
\hline 10 & 1078 & 0.0043 & 1077 & 0.0014 & 1077 & 0.0017 & 1077 & 0.0016 \\
\hline 11 & 1265 & 0.0132 & 1108 & 0.0010 & 1108 & 0.0010 & 1108 & 0.0011 \\
\hline 12 & 1286 & 0.0197 & 1264 & 0.0024 & 1263 & 0.0020 & 1256 & 0.0003 \\
\hline Ring wagging $+\mathrm{C}-\mathrm{C}$ & 1384 & 0.1820 & 1304 & 0.2712 & 1298 & 0.0690 & 1289 & 0.0006 \\
\hline stretch $+\mathrm{C}-\mathrm{H}$ bend & 1423 & 0.2330 & 1315 & 0.0001 & 1307 & 0.1391 & 1305 & 0.3148 \\
\hline 15 & 1458 & 0.0968 & 1377 & 0.3107 & 1372 & 0.4019 & 1366 & 0.4909 \\
\hline 16 & 1611 & 0.0142 & 1545 & 0.0286 & 1543 & 0.0292 & 1540 & 0.0367 \\
\hline 17 & 1678 & 0.0174 & 1617 & 0.0044 & 1617 & 0.0043 & 1617 & 0.0043 \\
\hline 18 & 3321 & 0.0034 & 2675 & 0.0224 & 2541 & 0.0165 & 2292 & 0.0106 \\
\hline 19 & 3334 & 0.0341 & 2722 & 0.0009 & 2585 & 0.0016 & 2330 & 0.0013 \\
\hline 20 & 3339 & 0.0004 & 3321 & 0.0034 & 3321 & 0.0034 & 3321 & 0.0034 \\
\hline 21 & 3394 & 0.0065 & 3339 & 0.0008 & 3339 & 0.0008 & 3339 & 0.0008 \\
\hline
\end{tabular}

Table 6 Calculated vibrational normal-mode frequencies $\omega$ (in $\mathrm{cm}^{-1}$ ) and corresponding Huang-Rhys factors $S$ (only for non-zero values) vary with respect to the scaling parameters for absorption spectrum in cyclohexane solvent. The scaling parameters $\zeta$ for 8 backbone- $\mathrm{H}$ atoms 15,16 , $17,18,23,24,25$, and 26 (see Fig. 1) are changed equally as listed below and for the rest of hydrogen atoms and all carbon atoms are fixed as $\zeta_{H}=$ 1.0 and $\zeta_{C}=1.0$ (no scaling)

\begin{tabular}{|c|c|c|c|c|c|c|c|c|}
\hline \multirow{2}{*}{$\begin{array}{l}\text { Total symmetry vibration } \\
\text { modes }\left(\mathrm{A}_{\mathrm{g}}\right)\end{array}$} & \multicolumn{2}{|c|}{$\zeta_{\mathrm{H}}=1.0$} & \multicolumn{2}{|c|}{$\zeta_{\mathrm{H}}=1.4$} & \multicolumn{2}{|c|}{$\zeta_{\mathrm{H}}=1.6$} & \multicolumn{2}{|c|}{$\zeta_{\mathrm{H}}=2.0$} \\
\hline & $\omega$ & $S$ & $\omega$ & $S$ & $\omega$ & $S$ & $\omega$ & $S$ \\
\hline 2 & 213 & 0.0216 & 210 & 0.0198 & 209 & 0.0197 & 206 & 0.0194 \\
\hline 3 & 275 & 0.0340 & 268 & 0.0342 & 264 & 0.0315 & 257 & 0.0323 \\
\hline 4 & 357 & 0.0971 & 351 & 0.0958 & 346 & 0.0944 & 340 & 0.1016 \\
\hline 7 & 772 & 0.0049 & 696 & 0.0201 & 688 & 0.0177 & 675 & 0.0139 \\
\hline 8 & 828 & 0.0054 & 777 & 0.0013 & 753 & 0.0006 & 712 & 0.0001 \\
\hline 9 & 1043 & 0.0214 & 985 & 0.0002 & 955 & 0.0001 & 871 & 0.0002 \\
\hline 10 & 1078 & 0.0044 & 1019 & 0.0035 & 979 & 0.0012 & 918 & 0.0144 \\
\hline 11 & 1267 & 0.0191 & 1139 & 0.0040 & 1056 & 0.0032 & 1038 & 0.0063 \\
\hline 12 & 1294 & 0.0061 & 1245 & 0.0027 & 1236 & 0.0059 & 1223 & 0.0074 \\
\hline \multirow{4}{*}{$\begin{array}{l}\text { Ring wagging }+\mathrm{C}-\mathrm{C} \\
\text { stretch }+\mathrm{C}-\mathrm{H} \text { bend }\end{array}$} & 1669 & 0.1360 & 1662 & 0.1493 & 1653 & 0.2429 & 1646 & 0.2473 \\
\hline & 3320 & 0.0039 & 2856 & 0.0047 & 2694 & 0.0010 & 2454 & 0.0012 \\
\hline & 3329 & 0.0342 & 2917 & 0.0019 & 2702 & 0.0164 & 2460 & 0.0070 \\
\hline & 3396 & 0.0065 & 2919 & 0.0023 & 2757 & 0.0018 & 2512 & 0.0010 \\
\hline
\end{tabular}

at $\mathrm{S}_{1}-D_{2 \mathrm{H}}$, there are three active normal modes $\left(v_{13}, v_{14}\right.$, and $\left.v_{15}\right)$ $=(1384,1423$, and 1458$) \mathrm{cm}^{-1}$ corresponding to $S$-value 0.182 , 0.233 , and 0.10 , respectively within benzene solvent and $\left(v_{12}\right.$, $v_{14}$ and $\left.v_{15}\right)=(1286,1384$, and 1423$) \mathrm{cm}^{-1}$ corresponding to $S$ value $0.187,0.185$, and 0.223 , respectively within cyclohexane solvent. All Huang-Rhys factors for $\mathrm{A}_{\mathrm{g}}$ modes are listed in no 
scaling $\left(\zeta_{\mathrm{H}}=1.0, \zeta_{\mathrm{C}}=1.0\right)$ column of Table 5 for benzene solvent and in Table 7 for cyclohexane solvent, three active normal modes all vibrates as ring wagging plus CC stretch and $\mathrm{CH}$ bend motion on the backbone as shown in Fig. S4(b) and S5(b) (ESI $\dagger$ ). We can immediately conclude that fluorescence spectrum is slightly different in non-polar benzene and polar cyclohexane solvents because the three active normal-mode motions are slightly different. However, Huang-Rhys factors for absorption spectra calculated with PCM in benzene solvent (see the first column of Table 4) and in cyclohexane solvent (see the first column of Table 6) are almost the same for corresponding vibrational frequencies. Huang-Rhys factors for fluorescence spectra calculated with PCM in benzene solvent (see the first column of Table 5) and in cyclohexane solvent (see the first column of Table 7) are also almost the same for corresponding vibrational frequencies. This actually reflects that PCM method can show the certain amount change for equilibrium geometries of ground and excited states individually, but change of geometry difference between ground and excited states is very small and thus it does not affect Huang-Rhys factors while passing from gaseous to solution phase. We checked this point by calculating Huang-Rhys factors in gaseous phase not only for $D_{2 \mathrm{H}}$ symmetry, but also for $D_{2}$ and $C_{2 \mathrm{H}}$ symmetries (the great detailed discussion for perylene in benzene solvent was made in ref. 36). We also notify that Huang-Rhys factors for absorption spectra (see the first column of Table 4) and for fluorescence spectra (see the first column of Table 5) are not big for corresponding vibrational frequencies, and this reflects that Duschinsky rotation effect is small.
The vibornic profiles including peaks and widths of absorption and fluorescence spectra are mostly determined by combination of four factors in Franck-Condon simulation: vibronic coupling distributions (Huang-Rhys factors), adiabatic excitation energies, inhomogeneous broadening of solvent effect for electronic part, and interaction between solute molecule and solvent molecules for nuclear part. There is an additional parameter called as homogeneous broadening (can be regarded as instrumental resolution in experiment), and it is taken as $\gamma_{\mathrm{ab}}=20 \mathrm{~cm}^{-1}$ in eqn (1) and (2) for simulating absorption and fluorescence spectra of rubrene with room temperature $T=298 \mathrm{~K}$. Inhomogeneous broadening parameters are chosen as $D_{\mathrm{ab}}=650 \mathrm{~cm}^{-1}\left(700 \mathrm{~cm}^{-1}\right)$ in eqn (1) and $D_{\mathrm{ab}}$ $=600 \mathrm{~cm}^{-1}\left(650 \mathrm{~cm}^{-1}\right)$ in eqn (2), respectively for simulating absorption and fluorescence spectra of rubrene in benzene (cyclohexane) solvent. The present choice of inhomogeneous broadening is because those observed widths of absorption and fluorescence from experiment are slightly broader in cyclohexane than in benzene solvent. Interaction between solute molecule and solvent molecules is represented by damped FC factor with the scaling parameters that are scaled slightly different in benzene and cyclohexane solvents. Therefore, we discuss separately in the following sub-sections.

\subsection{Absorption and fluorescence spectra in benzene solvent}

The Stokes shift (energy gap between the two peaks most close to adiabatic excitation energy $\omega_{\mathrm{ba}}$ in absorption and fluorescence spectra) is $1120 \mathrm{~cm}^{-1}, 1200 \mathrm{~cm}^{-1}$, and $1200 \mathrm{~cm}^{-1}$,

Table 7 Calculated vibrational normal-mode frequencies $\omega$ (in $\mathrm{cm}^{-1}$ ) and corresponding Huang-Rhys factors $S$ (only for non-zero values) vary with respect to the scaling parameters $\zeta$ for fluorescence spectra in cyclohexane solvent. The scaling parameters $\zeta$ for 8 backbone- $\mathrm{H}$ atoms plus 12 backbone-C atoms 1, 2, 3, 4, 5, and 6, and 12, 13, 19, 20, 21, and 22 (see Fig. 1) are varied as listed below and for the rest of hydrogen atoms and the rest of carbon atoms are fixed as $\zeta_{H}=1.0$ and $\zeta_{C}=1.0$ (no scaling)

\begin{tabular}{|c|c|c|c|c|c|c|c|c|}
\hline \multirow{2}{*}{$\begin{array}{l}\text { Total symmetry vibration } \\
\text { modes }\left(\mathrm{A}_{\mathrm{g}}\right)\end{array}$} & \multicolumn{2}{|c|}{$\zeta_{\mathrm{H}}=1.0, \zeta_{\mathrm{C}}=1.0$} & \multicolumn{2}{|c|}{$\zeta_{\mathrm{H}}=1.4, \zeta_{\mathrm{C}}=1.4$} & \multicolumn{2}{|c|}{$\underline{\zeta_{\mathrm{H}}}=1.6, \zeta_{\mathrm{C}}=1.4$} & \multicolumn{2}{|c|}{$\zeta_{\mathrm{H}}=1.9, \zeta_{\mathrm{C}}=1.4$} \\
\hline & $\omega$ & $S$ & $\omega$ & $S$ & $\omega$ & $S$ & $\omega$ & $S$ \\
\hline \multirow{22}{*}{$\begin{array}{l}\text { Ring wagging }+\mathrm{C}-\mathrm{C} \\
\text { stretch }+\mathrm{C}-\mathrm{H} \text { bend }\end{array}$} & 89 & 0.0160 & 89 & 0.0160 & 89 & 0.0160 & 89 & 0.0160 \\
\hline & 210 & 0.0238 & 210 & 0.0245 & 210 & 0.0245 & 210 & 0.0245 \\
\hline & 273 & 0.0348 & 257 & 0.0589 & 256 & 0.0587 & 255 & 0.0626 \\
\hline & 354 & 0.0994 & 321 & 0.0261 & 321 & 0.0253 & 320 & 0.0246 \\
\hline & 582 & 0.0120 & 573 & 0.0178 & 572 & 0.0192 & 570 & 0.0281 \\
\hline & 670 & 0.0138 & 588 & 0.0008 & 586 & 0.0003 & 584 & 0.0000 \\
\hline & 706 & 0.0139 & 687 & 0.0012 & 687 & 0.0026 & 686 & 0.0023 \\
\hline & 772 & 0.0054 & 772 & 0.0040 & 771 & 0.0046 & 771 & 0.0046 \\
\hline & 828 & 0.0076 & 818 & 0.0095 & 818 & 0.0088 & 817 & 0.0113 \\
\hline & 1059 & 0.0134 & 994 & 0.0001 & 982 & 0.0015 & 943 & 0.0107 \\
\hline & 1078 & 0.0044 & 1027 & 0.0046 & 1020 & 0.0013 & 1021 & 0.0030 \\
\hline & 1286 & 0.1870 & 1211 & 0.2921 & 1210 & 0.3282 & 1210 & 0.3192 \\
\hline & 1289 & 0.0039 & 1264 & 0.0012 & 1259 & 0.0056 & 1241 & 0.0246 \\
\hline & 1384 & 0.1853 & 1306 & 0.0089 & 1289 & 0.0002 & 1289 & 0.0003 \\
\hline & 1423 & 0.2226 & 1340 & 0.1774 & 1333 & 0.3066 & 1331 & 0.4001 \\
\hline & 1458 & 0.0979 & 1403 & 0.0020 & 1387 & 0.0002 & 1375 & 0.0048 \\
\hline & 1612 & 0.0141 & 1503 & 0.0015 & 1502 & 0.0035 & 1501 & 0.0038 \\
\hline & 1622 & 0.0384 & 1618 & 0.0044 & 1618 & 0.0043 & 1618 & 0.0043 \\
\hline & 3320 & 0.0034 & 2817 & 0.0264 & 2652 & 0.0302 & 2457 & 0.0206 \\
\hline & 3333 & 0.0343 & 2869 & 0.0055 & 2700 & 0.0034 & 2500 & 0.0009 \\
\hline & 3339 & 0.0001 & 3320 & 0.0034 & 3320 & 0.0034 & 3320 & 0.0034 \\
\hline & 3394 & 0.0065 & 3339 & 0.0007 & 3339 & 0.0007 & 3339 & 0.0007 \\
\hline
\end{tabular}


respectively from experimental measurement (see Fig. 5(a)), and the present simulation with scaling (see Fig. 5(b)) and without scaling (see Fig. 5(c)) in benzene solvent. Actually, simulated vibronic band peaks all agree with experimental results as shown in Fig. 5, and the simulated peaks do not depend on change of scaling parameters as shown in Fig. S6 and S7 (ESI $\dagger$ ). However, the intensity of the second maximum band peaks from simulation is much weaker than those from experiment for absorption spectrum if we compare Fig. 5(a) with Fig. 5(c), in fact, these solvent enhanced band shapes cannot be well reproduced without scaling. The first consideration about scaling is focused on all hydrogen atoms in rubrene as we did in ref. 36, however, there are distinct two group types of hydrogen atoms: one is 8 hydrogen atoms $(15,16,17,18,23,24,25$, and 26) on the backbone and the other is associated with four phenyl rings. The preliminary test was done by scaling all hydrogen atoms in the same way, and then we looked at how absorption spectrum varies as scaling parameters. We found there is little influence on spectrum by scaling those hydrogen atoms associated with four phenyl rings, while only scaling on 8 hydrogen atoms on the backbone makes sense of spectrum. This is actually consistent with analysis given in Subsection 3.1 about electronic excitation and molecular orbitals all related with the backbone atoms. Therefore, we work on only scaling 8 backbone-H atoms equally varying from $\zeta_{\mathrm{H}}=1.0,1.4,1.6$, and 2.0 (no-scaling on the other hydrogen atoms and all carbon atoms), and we see from Table 4 that the only $S$ values related to four active normal modes $\left(v_{15}, v_{16}, v_{17}\right.$, and $\left.v_{20}\right)=(1389,1416$, 1555, and 1669) $\mathrm{cm}^{-1}$ change, while the rest of $S$ values has no meaningful change. For instance, the $S$ values change from $0.356,0.050,0.037$, and 0.137 at $\zeta_{\mathrm{H}}=1.0$ (means no scaling) to $0.069,0.227,0.288$, and 0.247 at $\zeta_{\mathrm{H}}=2.0$ for the corresponding four active normal modes $\left(v_{15}, v_{16}, v_{17}\right.$, and $\left.v_{20}\right)$, respectively. Simulated absorption spectrum with scaling parameter at $\zeta_{\mathrm{H}}=$ 2.0 well reproduces solvent enhanced absorption spectrum as shown in Fig. 5(b), besides the present scaling scheme is very smooth and stable (just like in ref. 36) as shown in Table 4 and S6 (ESI $\dagger$ ) for absorption spectrum of rubrene molecule in benzene solvent.

For fluorescence spectrum, we can see from Fig. 5(a) and (c) that intensity enhancement is not as much as absorption spectrum for the second maximum band peak and actually the present simulation works to some extent even without scaling. However, fluorescence band shape is not well reproduced without scaling. We have done the preliminary test with only scaling 8 backbone-H atoms, but it did not work out for detailed band shape. Therefore we add scaling on 12 carbon atoms (1, 2, 3, 4, 5, 6, 12, 13, 19, 20, 21 and 22) at two ends of the backbone, so that we make scaling on these 12 carbon atoms equally denoted as $\zeta_{\mathrm{C}}=$ and on the 8 backbone- $\mathrm{H}$ atoms as $\zeta_{\mathrm{H}}=$, and the rest of $\mathrm{H}$ and $\mathrm{C}$ atoms are kept no scaling. In this way, simulated fluorescence spectrum with combination scaling $\zeta_{\mathrm{H}}=2.3$ and $\zeta_{\mathrm{C}}=1.2$ can reproduce detailed band shapes as shown in Fig. 5(b). We see from Table 5 that the only $S$ values related to three active normal modes $\left(v_{13}, v_{14}\right.$, and $\left.v_{15}\right)$ $=(1384,1423$, and 1458$) \mathrm{cm}^{-1}$ change, and the rest of $S$ values has no meaningful change. For example, the $S$ values change from $0.182,0.233$, and 0.097 at $\zeta_{\mathrm{H}}=1.0$ and $\zeta_{\mathrm{C}}=1.0$ (means no scaling) to $0.0006,0.315$, and 0.491 at $\zeta_{\mathrm{H}}=2.3$ and $\zeta_{\mathrm{C}}=1.2$ for the corresponding three active normal modes $\left(v_{13}, v_{14}\right.$, and $v_{15}$ ), respectively. Table 5 and Fig. S7 (ESI $\dagger$ ) again show that the present scaling scheme is very smooth and stable for fluorescence spectrum of rubrene molecule in benzene solvent.

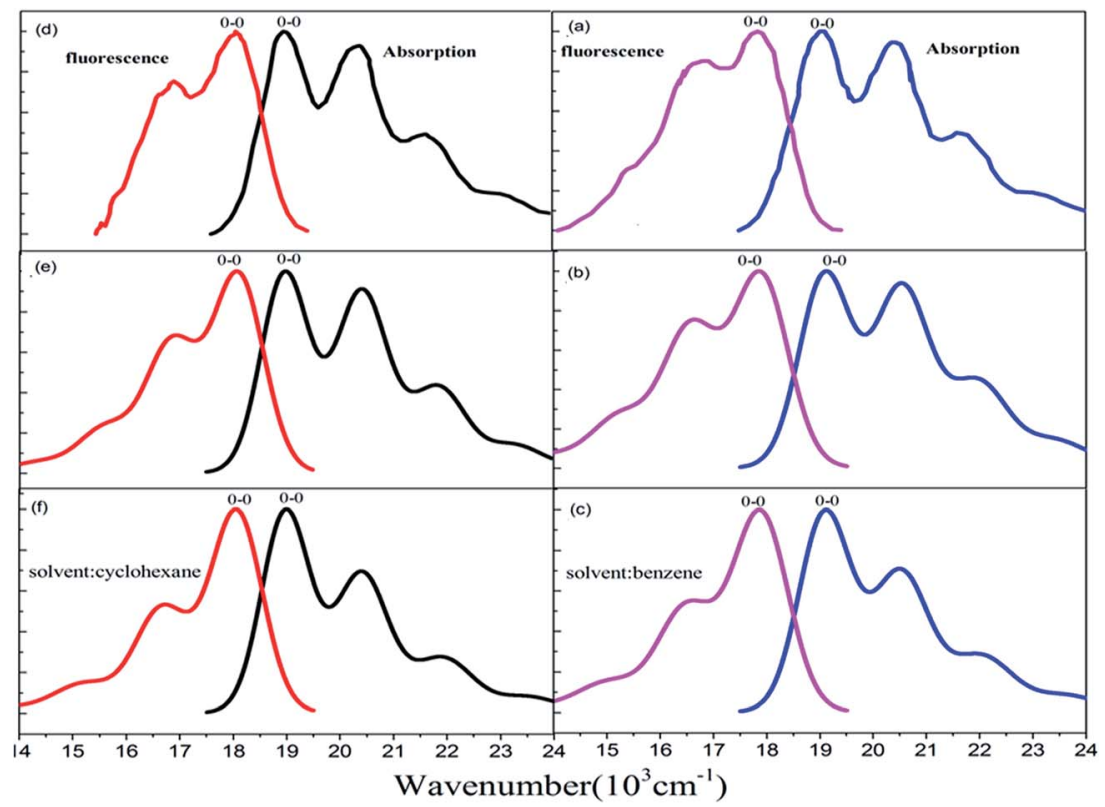

Fig. 5 Measured and calculated $S_{0}\left({ }^{1} A_{g}\right) \rightarrow S_{1}\left({ }^{1} B_{3 u}\right)$ absorption and $S_{0}\left({ }^{1} A_{g}\right) \leftarrow S_{1}\left({ }^{1} B_{3 u}\right)$ fluorescence spectra at temperature $T=298 \mathrm{~K}$. (a) Experiment from ref. 43, simulated (b) with and (c) without scaling in benzene solvent. (d) Experiment from ref. 42, simulated (e) with and (f) without scaling in cyclohexane solvent. 


\subsection{Absorption and fluorescence spectra in cyclohexane solvent}

In cyclohexane solvent, the Stokes shift is $820 \mathrm{~cm}^{-1}, 830 \mathrm{~cm}^{-1}$, and $830 \mathrm{~cm}^{-1}$, respectively from experimental measurement (see Fig. 5(d)), and the present simulation with scaling (see Fig. 5(e)) and without scaling (see Fig. 5(f)). Actually, all simulated peak positions in vibronic spectral bands agree with experimental results as shown in Fig. 5, and again simulated vibronic peaks do not depend on change of scaling parameters as shown in Fig. S8 and S9 (ESI $\dagger$ ). For absorption spectrum in cyclohexane solvent, we work on only scaling 8 backbone- $\mathrm{H}$ atoms equally varying from $\zeta_{\mathrm{H}}=1.0,1.4,1.6$, and 2.0 (like we did in benzene solvent), and we see from Table 6 that the only $S$ values vary the almost same as those in Table 4 for four active normal modes $\left(v_{13}, v_{14}, v_{15}\right.$, and $\left.v_{17}\right)=(1390,1415,1555$, and 1669) $\mathrm{cm}^{-1}$. Simulated absorption spectrum with scaling parameter at $\zeta_{\mathrm{H}}=2.0$ well reproduces solvent enhanced absorption spectrum in cyclohexane solvent as shown in Fig. 5(e).

For fluorescence spectrum in cyclohexane solvent, we do the same scaling as in benzene solvent for the 8 backbone- $\mathrm{H}$ atoms and 12 backbone-C atoms, and as a result, simulated fluorescence spectrum with combination $\zeta_{\mathrm{H}}=1.9$ and $\zeta_{\mathrm{C}}=1.4$ can well reproduce detailed band shapes as shown in Fig. 5(e). Table 7 shows that the only $S$ values related to three active normal modes $\left(v_{12}, v_{14}\right.$ and $\left.v_{15}\right)=\left(1286,1384\right.$, and 1423) $\mathrm{cm}^{-1}$ change and the rest of $S$ values has no meaningful change. Fig. S8 and S9 (ESI $\dagger$ ) confirm that vibronic spectra vary very smoothly with respect to change of scaling parameters in cyclohexane solvent.

\section{Concluding remark}

We have employed four types of density functionals such as (TD)-B3LYP, (TD)-B3LYP35, (TD)-B3LYP50, and HF-CIS plus PCM models to investigate topology of potential energy surfaces on the ground state $S_{0}$ and the first excited state $S_{1}$ for rubrene. Geometry optimization calculations with the four-type functionals have revealed that there are four pairs of the local minima, $\mathrm{S}_{0}-D_{2}(1)$ and $\mathrm{S}_{1}-D_{2}(1), \mathrm{S}_{0}-D_{2}(2)$ and $\mathrm{S}_{1}-D_{2}(2), \mathrm{S}_{0}-C_{2 \mathrm{H}}(1)$ and $S_{1}-C_{2 H}(1)$, and $S_{0}-C_{2 H}(2)$ and $S_{1}-C_{2 H}(2)$, and one pair of the second-order transition state $\mathrm{S}_{0}-D_{2 \mathrm{H}}$ and $\mathrm{S}_{1}-D_{2 \mathrm{H}}$ that connect four local minima on $S_{0}$ and $S_{1}$ potential energy surfaces, respectively. The vibronic coupling distributions (Huang-Rhys factors) based on each pair of frequency calculations only support that the second-order transition state $\left(\mathrm{S}_{0}-D_{2 \mathrm{H}}\right.$ and $\mathrm{S}_{1}$ $D_{2 \mathrm{H}}$ ) can reproduce experimentally observed absorption and fluorescence spectra, and we have actually demonstrated that (TD)-BHandHLYP performs best for FC spectral simulation for rubrene molecule in benzene and cyclohexane solvents. The Stokes shift $1120 \mathrm{~cm}^{-1}\left(820 \mathrm{~cm}^{-1}\right)$ and vibronic-band peak positions in absorption and fluorescence spectra in non-polar benzene (polar cyclohexane) solvent are well reproduced within the displaced harmonic oscillator FC simulation (no need to have damped correction). However, the second strongest vibronic-band peak intensities and shapes in both absorption and fluorescence spectra are not in good agreement with experiment results without the scaling, so that the damped FC simulation comes to apply to resolve this deviation. We found that only hydrogen atoms and carbon atoms associated with the aromatic backbone are sensitive to the scaling, while the hydrogen atoms and carbon atoms associated with four phenyl rings are not. Especially, we have shown in both absorption and fluorescence spectra that four vibrational modes around $(1390,1400,1550$, and 1670$) \mathrm{cm}^{-1}$ are responsible for solvent enhanced absorption spectra and three vibrational modes around $\left(1290,1380\right.$, and 1420) $\mathrm{cm}^{-1}$ are responsible for solvent enhanced fluorescence spectra. Those active modes all correspond to ring wagging plus CC stretch and $\mathrm{CH}$ bend motions of the atoms on the backbone, vibartional normal-mode motions associated with atoms on four phenyl rings are not active for vibronic spectra. Therefore, in the present work we have presented new physical insight how vibrational motions related to the aromatic backbone with $D_{2 \mathrm{H}}$ group symmetry play for interpreting solvent enhanced vibronic spectra of rubrene.

\section{Acknowledgements}

This work is supported by Ministry of Science and Technology of the Republic of China under grant no. 103-2113-M-009-007MY3 and National Natural Science Foundation of P. R. China under grant no. 21673085. S.-H. L thanks supports from Ministry of Science and Technology of the Republic of China under grant no. 105-2923-M-009-003. Y. H. thanks support from visiting student fellowship by National Chiao Tung University. C. Z. thanks the MOE-ATU project of the National Chiao Tung University for support.

\section{References}

1 S. H. Lin, C. H. Chang, K. K. Liang, R. Chang, Y. J. Shiu, J. M. Zhang, T. S. Yang, M. Hayashi, and F. C. Hsu, in Adv. Chem. Phys., John Wiley \& Sons, Inc., 2002, pp. 1-88.

2 C. Zhu, K. K. Liang, M. Hayashi and S. H. Lin, Chem. Phys., 2009, 358, 137-146.

3 H. Hwang and P. J. Rossky, J. Phys. Chem. A, 2004, 108, 26072616.

4 P. Malmqvist and N. Forsberg, Chem. Phys., 1998, 228, 227240.

5 F. T. Chau, J. M. D. Edmond, P. F. Lee and D. C. Wang, J. Electron Spectrosc. Relat. Phenom., 1998, 97, 33-47.

6 E. V. Doktorov, I. A. Malkin and V. I. Manko, J. Mol. Spectrosc., 1977, 64, 302-326.

7 S.-Y. Lee, J. Phys. Chem., 1990, 94, 4420-4425.

8 H. Kikuchi, M. Kubo, N. Watanabe and H. Suzuki, J. Chem. Phys., 2003, 119, 729.

9 L. Yang, C. Zhu, J. Yu and S. H. Lin, Chem. Phys., 2012, 400, 126-136.

10 T.-W. Huang, L. Yang, C. Zhu and S. H. Lin, Chem. Phys. Lett., 2012, 541, 110-116.

11 T. Petrenko and F. Neese, J. Chem. Phys., 2007, 127, 164319.

12 J. M. Luis, D. M. Bishop and B. Kirtman, J. Chem. Phys., 2004, 120, 813. 
13 F. J. A. Ferrer, V. Barone, C. Cappelli and F. Santoro, J. Chem. Theory Comput., 2013, 9, 3597-3611.

14 J. Lerme, Chem. Phys., 1990, 145, 67-88.

15 M. Roche, Chem. Phys. Lett., 1990, 168, 556-558.

16 A. Baiardi, J. Bloino and V. Barone, J. Chem. Theory Comput., 2013, 9, 4097-4115.

17 T. E. Sharp and H. M. Rosenstock, J. Chem. Phys., 1964, 41, 3453-3464.

18 H. Nagae, J. Chem. Phys., 1997, 106, 5159.

19 D. H. L. Linda and A. Peteanu, J. Phys. Chem., 1988, 92, 65546561.

20 I. Baraldi, G. Brancolini, F. Momicchioli, G. Ponterini and D. Vanossi, Chem. Phys., 2003, 288, 309-325.

21 Y. H. Wang, M. Halik, C. K. Wang, S. R. Marder and Y. Luo, J. Chem. Phys., 2005, 123, 194311-194331.

22 H. C. Georg, K. Coutinho and S. Canuto, J. Chem. Phys., 2007, 126, 034507-034510.

23 I. F. Galvan, M. E. Martin, A. Munoz-Losa, M. L. Sanchez and M. A. Aguilar, J. Chem. Theory Comput., 2011, 7, 1850-1857.

24 O. Clemens, M. Basters, M. Wild, S. Wilbrand, C. Reichert, M. Bauer, M. Springborg and G. Jung, J. Mol. Struct.: THEOCHEM, 2008, 866, 15-20.

25 J. Zeng, N. S. Hush and J. R. Reimers, J. Chem. Phys., 1993, 99, 1508.

26 T. Sakata, Y. Kawashima and H. Nakano, J. Chem. Phys., 2011, 134, 014501.

27 O. V. Prezhdo, W. B. Valentyna, V. Zubkova and V. V. Prezhdo, J. Phys. Chem. A, 2008, 112, 13263-13266.

28 K. S. Schweizer and D. Chandler, J. Chem. Phys., 1983, 78, 4118-4125.

29 D. C. Tranca and A. A. Neufeld, J. Chem. Phys., 2009, 130, 141102.

30 A. N. Malakhov and A. L. Pankratov, in Adv. Chem. Phys., John Wiley \& Sons, Inc., 2002, pp. 357-438.

31 H. J. Kim, J. Chem. Phys., 1996, 105, 6833.

32 Y. Shigemitsu, M. Uejima, T. Sato, K. Tanaka and Y. Tominaga, J. Phys. Chem. A, 2012, 116, 9100.
33 W. Y. So, J. Hong, J. J. Kim, G. A. Sherwood, K. ChaconMadrid, J. H. Werner, A. P. Shreve, L. A. Peteanu and J. Wildeman, J. Phys. Chem. B, 2012, 116, 10504-10513.

34 F. Santoro, R. Improta, A. Lami, J. Bloino and V. Barone, J. Chem. Phys., 2007, 126, 084509.

35 M. Dracinsky and P. Bour, J. Chem. Theory Comput., 2010, 6, 288-299.

36 C. W. Wang, L. Yang, C. Zhu, J. Yu and S. H. Lin, J. Chem. Phys., 2014, 141, 084106.

37 H. Najafov, I. Biaggio, V. Podzorov, M. F. Calhoun and M. E. Gershenson, Phys. Rev. Lett., 2006, 96, 056604.

38 N. Sai, M. L. Tiago, J. R. Chelikowsky and F. A. Reboredo, Phys. Rev. B: Condens. Matter Mater. Phys., 2008, 77, 161306.

39 S. Tavazzi, A. Borghesi, A. Papagni, P. Spearman, L. Silvestri, A. Yassar, A. Camposeo, M. Polo and D. Pisignano, Phys. Rev. B: Condens. Matter Mater. Phys., 2007, 75, 245416.

40 C. Udhardt, R. Forker, M. Gruenewald, Y. Watanabe, T. Yamada, T. Ueba, T. Munakata and T. Fritz, Thin Solid Films, 2016, 598, 271-275.

41 L. Carmichael and G. L. Hug, Radiat. Phys. Chem., 1985, 26, 229-246.

42 T. Petrenko, O. Krylova, F. Neese and M. Sokolowski, New J. Phys., 2009, 11, 015001.

43 S. Tavazzi, L. Silvestri, M. Campione, A. Borghesi, A. Papagni, P. Spearman, A. Yassar, A. Camposeo and D. Pisignano, J. Appl. Phys., 2007, 102, 023107.

44 S. Chang, N. B. Rex and R. K. Chang, J. Opt. Soc. Am. B, 1999, 16, 1224-1235.

45 G. M. Badger and R. S. Pearce, Spectrochim. Acta, 1951, 4, 280-288.

46 F. Gao, W. Z. Liang and Y. Zhao, J. Phys. Chem. A, 2009, 113, 12847.

47 A. D. Becke, Phys. Rev. A, 1988, 38, 3098-3100.

48 H. C. Corben and P. Stehle, Classical Mechanics, Dover Publication, Inc., New York, 2nd edn, 1994.

49 M. J. Frisch, G. W. Trucks and H. B. Schlegel, Gaussian 09, Revision C.01, Gaussian, Inc., Wallingford, CT, 2010.

50 Q. Peng, Y. Yi, Z. Shuai and J. Shao, J. Am. Chem. Soc., 2007, 129, 9333. 